\title{
ESTRUCTURA INTERNA \\ Y DIFERENCIACION SOCIAL URBANA: COMUNA DE LA REINA
}

\author{
INTERNAL STRUCTURE AND URBAN SOCIAL DIFFERENTIATION: \\ "COMUNA LA REINA"
}

\author{
ELIANA FRANCO DE LA JARA Y JORGE ORTIZ VELIZ
}

Departamento de Geografia Universidad de Chile

\begin{abstract}
Land value and the role of the private management have been traditionally considered as explanatory forces of the socio-spatial differenciaton of the urban environment. However, in Chilean cities - specially those which have had a growing metropolization process - shouldbe added state's influence through housing programs for middle and low social sectors, some of them do not having access to formal housing market.

Three patterns of social differenciation were identified, starting from size, quality, state of maintenance, building year, land value, state role and action of neighboring groups: a) Consolidated urban area, characterized by its social heterogeneity; b/ transitional-expansional urban area, defined by its high status of social homogeneity, and cl peripheral-consolidated urban area of low economical status.

Special emphasis is put on the third category, which has been called subintegrated habitat. In fact, in front of the apparent homogeneity of this sector, it is possible indetify some social differences, specially from the point of view of the works status of the inhabitants, which are a product of the housing problem management in this space.
\end{abstract}

\section{ANTECEDENTES Y OBJETIVOS}

Los estudios de estructura intraurbana desarrollados a partir de la década del 20 del presente siglo, y que se inscriben en el marco de la ecología humana, centran fundamentalmente su interés en uno de los componentes del análisis urbano: la dimensión social. Los patrones espaciales resultantes, tienen como base explicativa los valores cambiantes del suelo y el grado de movilidad espacial de la población con acceso al mercado formal de la vivienda. De esta forma, tales patrones, corresponden a una politica económica en la que el mercado y la iniciativa privada surgen como las principales fuerzas organizativas del medio urbano. Al respecto, para una discusión más exhaustiva de los aspectos teóricos como metodológicos, existe una amplia 
literatura editada por geógrafos a la cual el lector puede remitirse (BERRY, 1975, 1977; HERBERT, 1972, 1979; HERBERT Y JOHNSTON, 1978).

En el caso de las ciudades chilenas, especialmente de aquellas que han vivido un proceso de concentración industrial y de metropolización, se agregan a estas fuerzas otros factores. En primer lugar, el papel desempeñado por el Estado a través de programas de construcción de vivienda para los sectores sociales medios y bajos; en segundo lugar, la existencia de grupos poblacionales sin acceso al mercado formal, quienes han generado como respuesta una ocupación espontánea en áreas periféricas; finalmente, y en estrecha relación con los factores anteriormente señalados, está presente el excesivo crecimiento demográfico, producto tanto de las altas tasas de incremento natural como también del proceso migratorio a los centros supuestamente más activos desde el punto de vista de la producción urbana. De esta forma, la movilidad residencial, fruto de una libre decisión individual, se presenta como un proceso fuertemente constreñido por factores externos al condicionamiento del mercado, si la comparamos con otras realidades.

En relación a este problema, se propone detectar y explicar la organización del espacio social en el Area Metropolitana de Santiago, tanto en su funcionamiento interno como en cuanto a la participación de la estructura decisional en la generación de tales patrones.

El presente trabajo representa un avance de dicho proyecto en la Comuna de La Reina, entidad espacial donde se ha seleccionado un conjunto de variables necesarias para el enfrentamiento de este tipo de estudios y los pasos metodológicos correspondientes, a fin de ser implementado posteriormente al resto del Area Metropolitana. Partiendo de la base de que se trata de un área básicamente residencial, los patrones de diferenciación se han establecido para cada manzana a partir de la evaluación de las caracteristicas físicas de la vivienda en términos de tamaño, calidad, estado de conservación, año de construcción, además de considerar el valor del suelo urbano.

Las explicaciones estructurales en este microespacio estan dadas por una variedad de aspectos que inciden en el valor del suelo urbano: accesibilidad, vecindad, externalidades, monopolio en la demanda, junto a un factor que rompe un tanto la "racionalidad de los elementos económicos", como es la acción directa del Estado en la conformación voluntaria de espacios socialmente segregados a través de diversas modalidades operativas.

El paso siguiente se circunscribe al estudio de espacios segregados, a fin de detectar su articulación tanto interna como externa. A tal efecto, se hace imprescindible en una etapa posterior, un análisis de tipo conductual de la población, en la medida de que se parte del supuesto que la segregación espacial de ella, genera estructuras funcionales diferentes al interior del espacio urbano, de acuerdo al status cultural, social, económico y a las formas de percibir el espacio realmente "vivido" (BUTIMER, 1982). En este punto interesa privilegiar aquellos sectores poblacionales de la Comuna asentados en áreas de mayor deterioro físico, enfatizando la dimensión espacial del "hábitat subintegrado" en su grado de funcionalidad de dicha condición. En este sentido, parece justo el planteamiento de SABATINI (1982: 54) al señalar que "la pobreza no es sólo un problema económico, social o cultural, sino también ambiental". El mismo autor expresa, que se "desconocen cómo estas características generan particulares formas de adaptación frente a la adversidad externa". La vecindad como formas de adscripción social; la economía "informal" interna como alternativa ocupacional o como mercado de bienes o servicios ofrecidos por los grupos familiares; el uso de la vivienda como fuente de ingreso, etc., al parecer, surgen como respuesta a tal adversidad.

Por otro lado, frente a la aparente homogeneidad de este tipo de hábitat, es posible identificar ciertas diferenciaciones de contenido social, producto de las modalidades o tipos de soluciones habitacionales originadas al interior de dichos espacios. En efecto, el origen de los asentamientos, el carácter de legalidad o transitoriedad de la propiedad, los requisitos necesarios para optar a ellas, implica también un cierto grado de desigualdad tanto económica como cultural, aspectos que son considerados en el presente trabajo.

\section{PRESENTACION DE LA COMUNA}

Creada a comienzos de la década del 60, la Comuna de La Reina corresponde a las necesidades de readecuación político administrativo de una metrópoli en expansión. En efecto, dependiendo en sus origenes de la antigua Comuna de Ñuñoa, tanto en la localización de una serie de equipamiento cívico-militar, así como un creciente proceso de parcelación de chacras y fundos agrícolas, donde se instalan sectores sociales de altos ingresos, llevaron a las autoridades a crear una Comuna independiente el 23 de febrero de 1963. En el fondo, la razón llevaba implicita vincular 


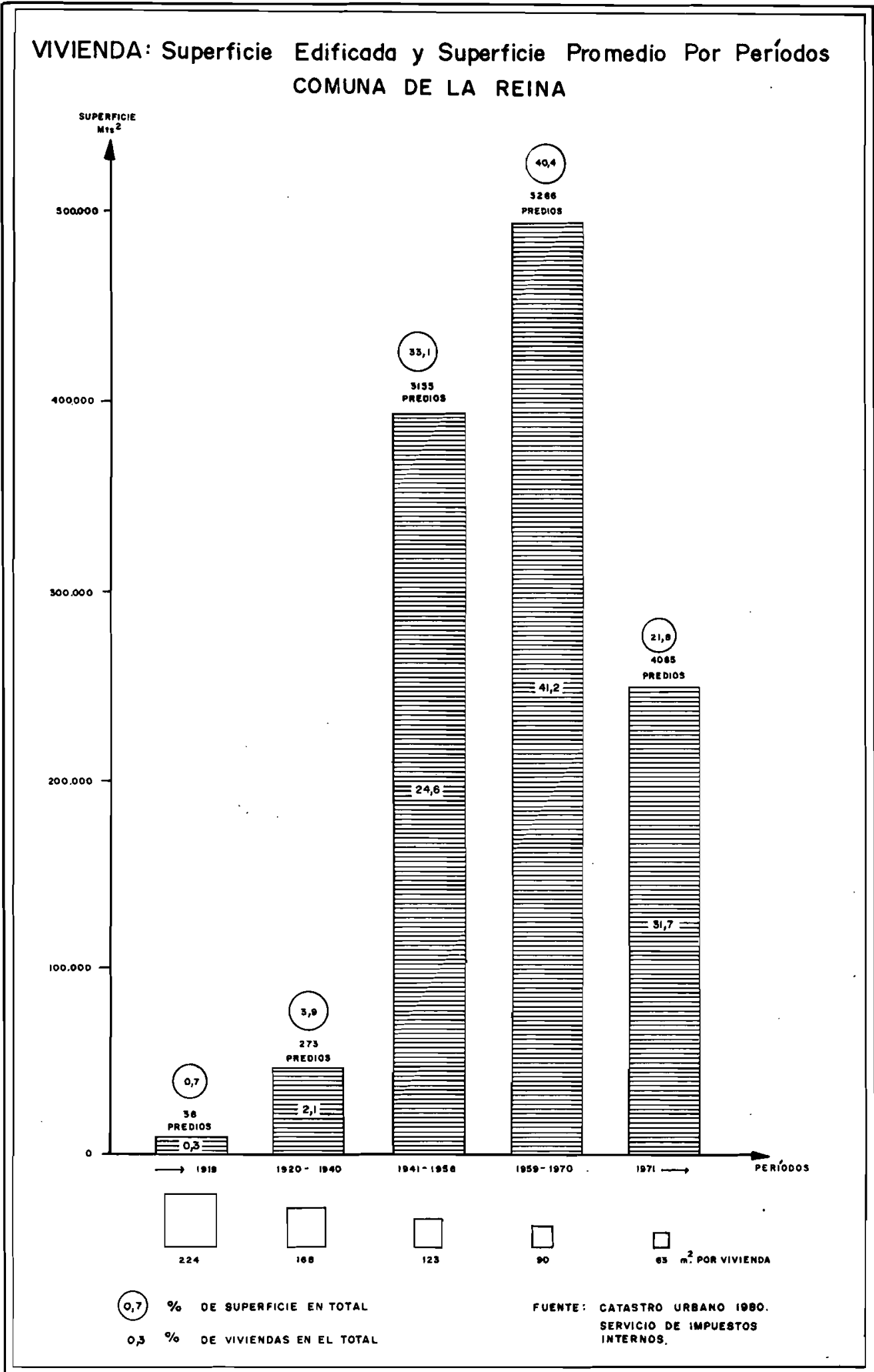

Fig. 1. 
este espacio al sector oriente del Area Metropolitana, adscrito a las Comunas de Providencia y Las Condes, conformando un sector geográficamente homogéneo desde el punto de vista socioeconómico. Por esta razón, la Comuna asume la categoría de sector de altos ingresos que por otra parte, la diferenciaría de su espacio original, - la Comuna de Nuñoa- sector tradicional de estratos medios.

El territorio comunal se eleva uniformemente hacia el piedmont y adopta una posición de "mirador" hacia Santiago. En la calle Alvaro Casanova alcanza la cota del Cerro San Cristóbal (866 m.s.n.m.). Este hecho, junto a su función predominantemente residencial, de vivienda unifamiliar de mediana densidad, ha materializado la imagen de "Comuna Verde" (DURÁN, 1982).

El último censo demográfico otorga una población de 79.631 habitantes (Instituto Nacional de Estadísticas, 1982) cifra que estuvo muy por debajo de las proyecciones estimadas que esperaban un total aproximado de 120.000 habitantes. En estas circunstancias, fue la única comuna de la Región Metropiltana que observó un crecimiento inferior al esperado. El alza considerable del valor del suelo en el área de expansión y la construcción de viviendas de lujo, desvió la localización de los sectores medios hacia otras comunas del Area Metropolitana.

En efecto, las inversiones privadas en construcción se localizan principalmente en Providencia y Las Condes, que concentran al año 1981 el $47 \%$ del total, en contraste a 1969, que representó el $26 \%$. Las Comunas de Ñuñoa y La Reina descienden su participación en el mismo período de un $21,4 \%$ al $19,4 \%$, en cambio, una nueva comuna de escaso crecimiento, La Florida, incrementa ostensiblemente su participación en materia de inversión privada en vivienda, (del $2 \%$ asciende al $21,4 \%)$. Las ofertas de viviendas en este sector ha tenido el sello publicitario de "la nueva comuna de los sectores medios".

Por este motivo, las transformaciones en materia de oferta y demanda, desde el punto de vista espacial, permiten explicar de alguna manera este "vacío demográfico" observado en el último censo en la Comuna de La Reina. En la actualidad, lujosas viviendas, recientemente construidas son ofertadas por el mercado conformando un sector de status socioeconómicos elevado, en el área cercana al piedmont como un proceso de expansión lateral del área oriente tradicional.

La ocupación residencial del territorio comunal, se define básicamente por presentar un desarrollo reciente. En efecto, de las 12.817 viviendas al año 1980 (Servicios de Impuestos Internos, 1980), el $73 \%$ corresponde a edificaciones posteriores a 1960 , concretadas especialmente en las áreas de transición -expansión y periférica- consolidada, y que en términos de superficie construida representa el $62 \%$ del total comunal. Por otro lado, en relación al tamaño promedio de la vivienda, aparece como significativo el decrecimiento de ella hacia los últimos años, coincidiendo las construcciones más antiguas (anteriores a 1960) con superficies superiores a $160 \mathrm{~m}^{2}$. (Fig. 1). En la actualidad, antiguas casaquintas y parcelas de agrado se constituyen en espacios relictos de la expansión urbana, especialmente en el sector de transición, testimoniando el pasado agrícola del área.

En síntesis, la comuna dista mucho de ser un espacio homogéneo. Diferencias en las etapas de crecimiento urbano, acción de ciertos umbrales que han actuado como barreras a la expansión, heterogeneidad en la estructura vial, diferenciación en la densidad habitacional, junto a la evaluación de las características físicas de la vivienda, han llevado a reconocer tres áreas al interior de ella. (Fig. 2) (Figs. 3, 4 y 5).

\section{CARACTERIZACION DE LA ESTRUCTURA INTERNA DE LA COMUNA}

\subsection{AREA URBANA CONSOLIDADA:}

Comprende el sector poniente de la Comuna. Es el área más antigua y centro del principal núcleo comercial de la Comuna. Está constituido por una red vial consolidada, donde destacan los principales ejes de comunicación tanto intercomunales como metropolitanas.

Esta área se caracteriza por su heterogeneidad, si se le compara con las dos restantes. Desde el punto de vista de los periodos de edificación de la vivienda no se observa un patrón claramente definido, producto del proceso de renovación y subdivisión predial a que ha estado sometida gracias a las externalidades positivas mencionadas con anterioridad.

El Sector Norte de esta área, se caracteriza por el predominio de viviendas de calidad media-superior, con superficies mayores a $100 \mathrm{~m}^{2}$, y valores del suelo más altos, o bien, donde se correlaciona estrechamente el valor de la construcción con el valor del terreno. En contraste, en el sector Sur se alcanzan valores más bajos del suelo urbano, una mayor densidad predial por 


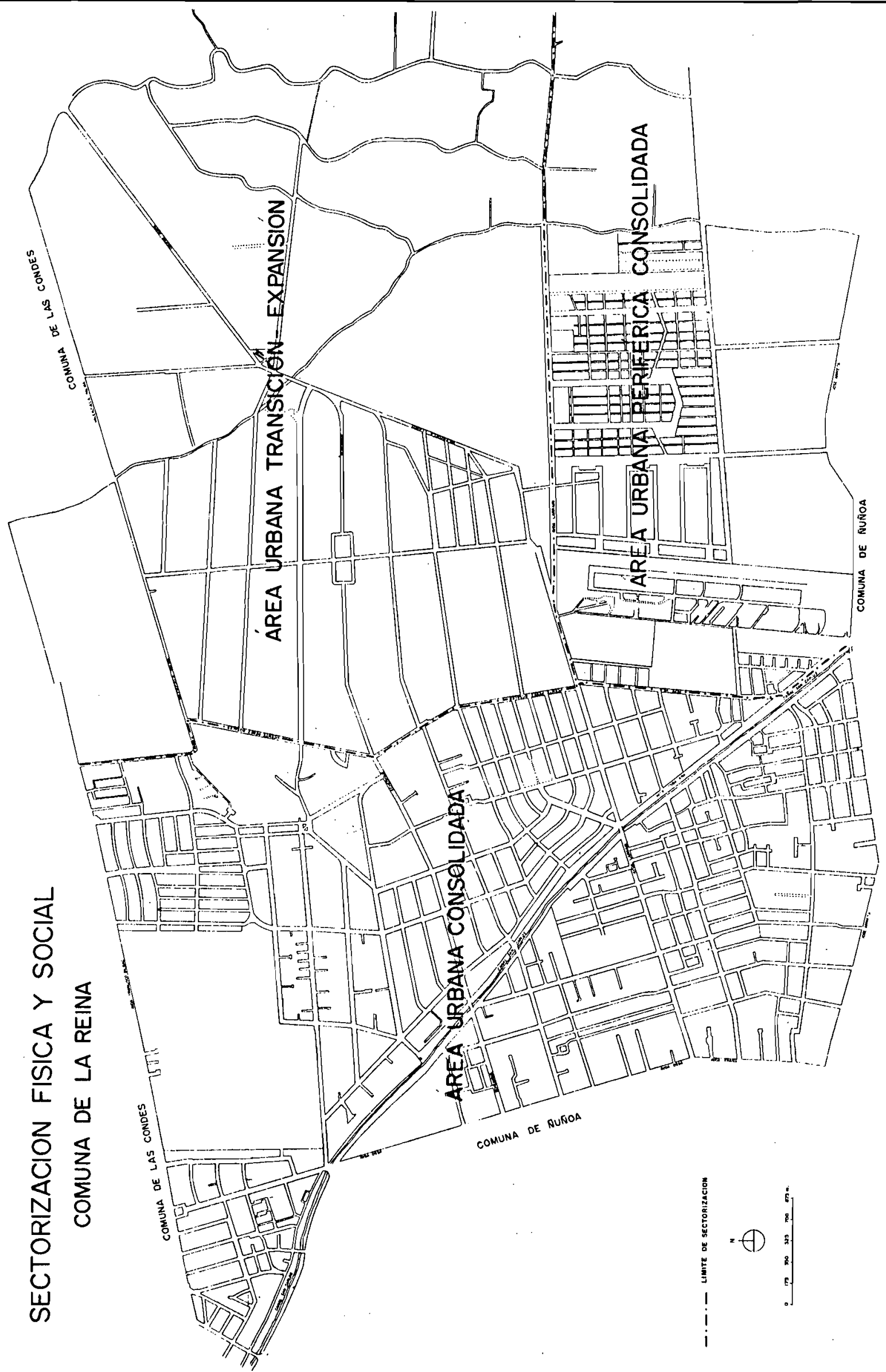




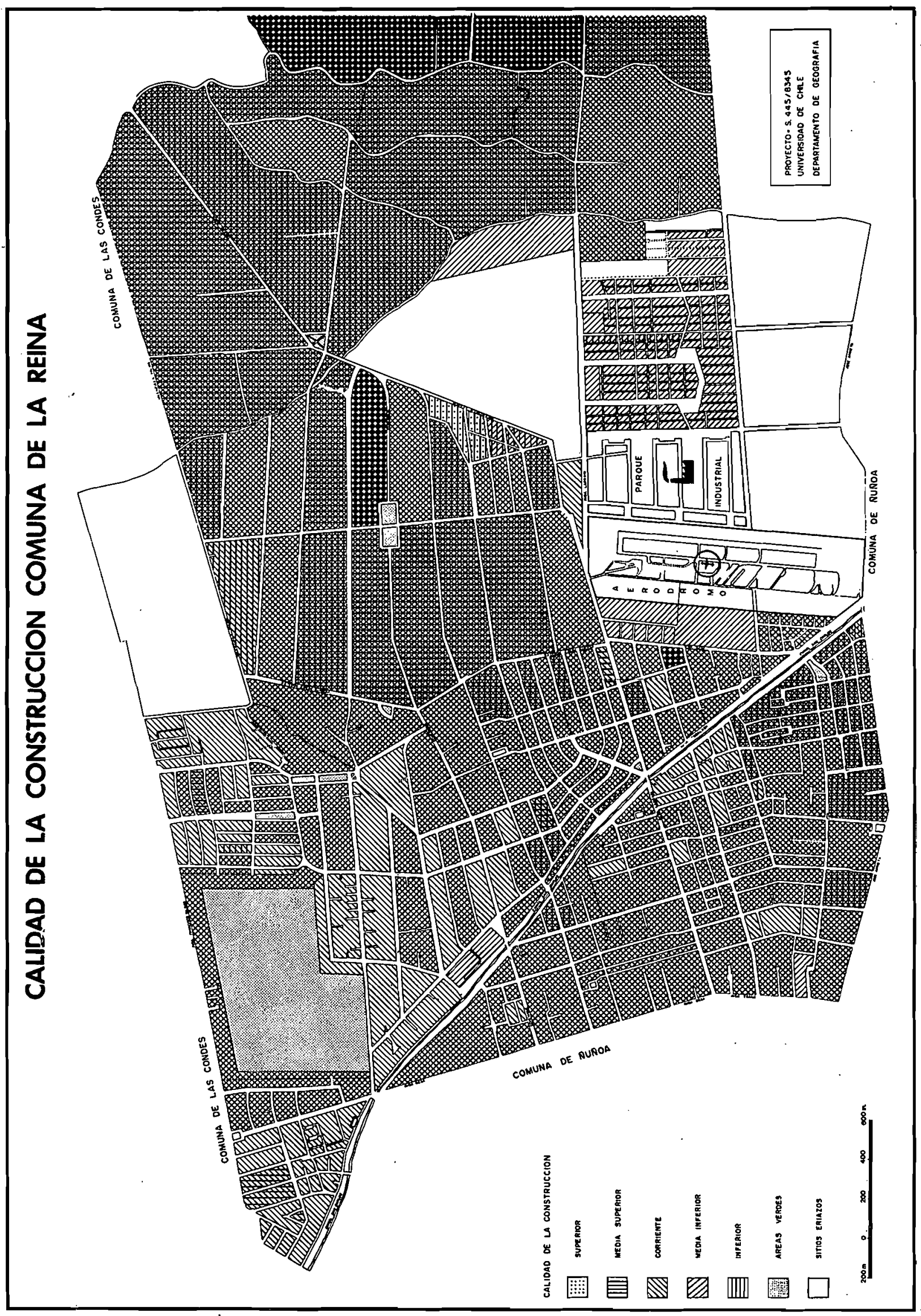




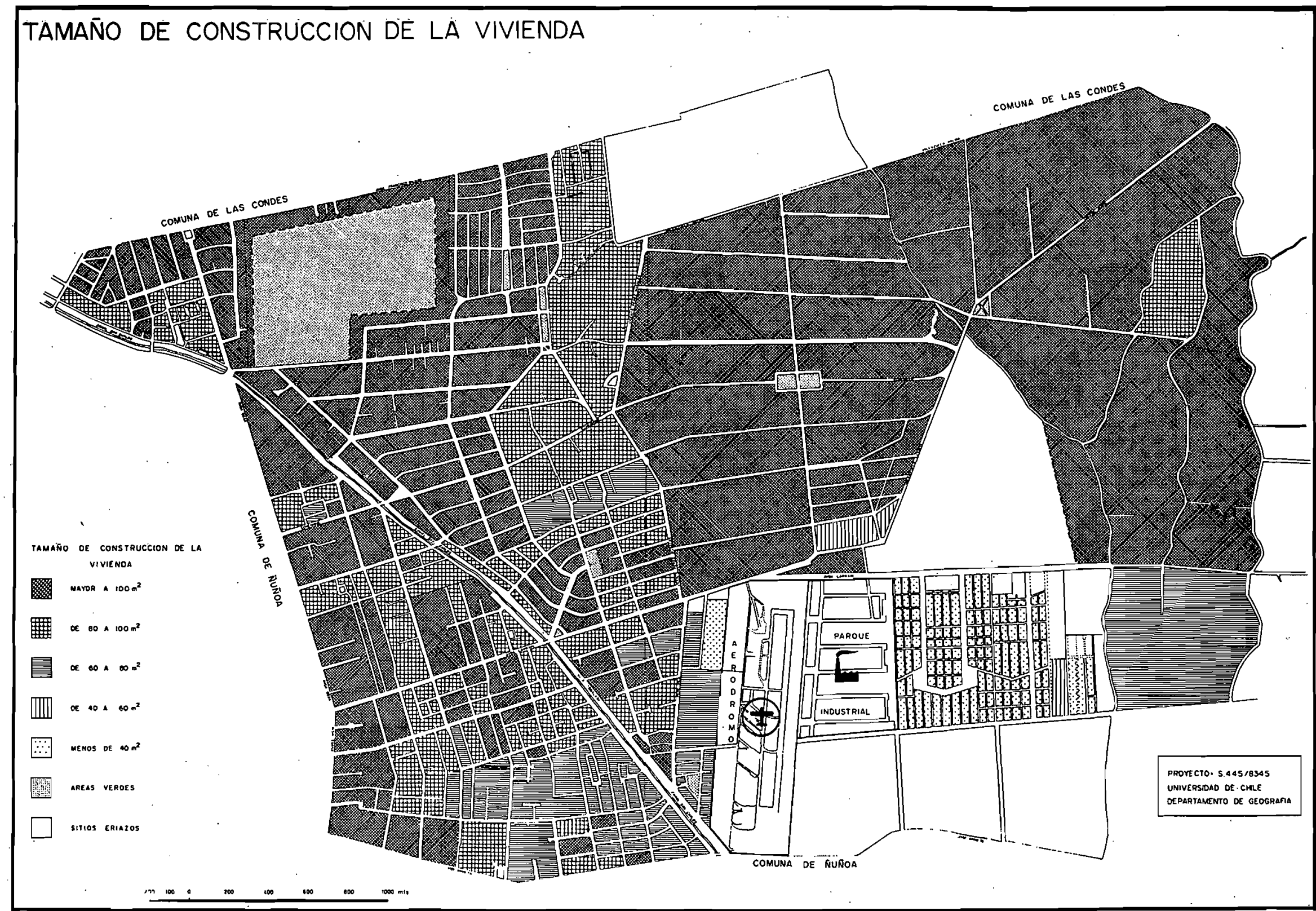

Fig. 4. 


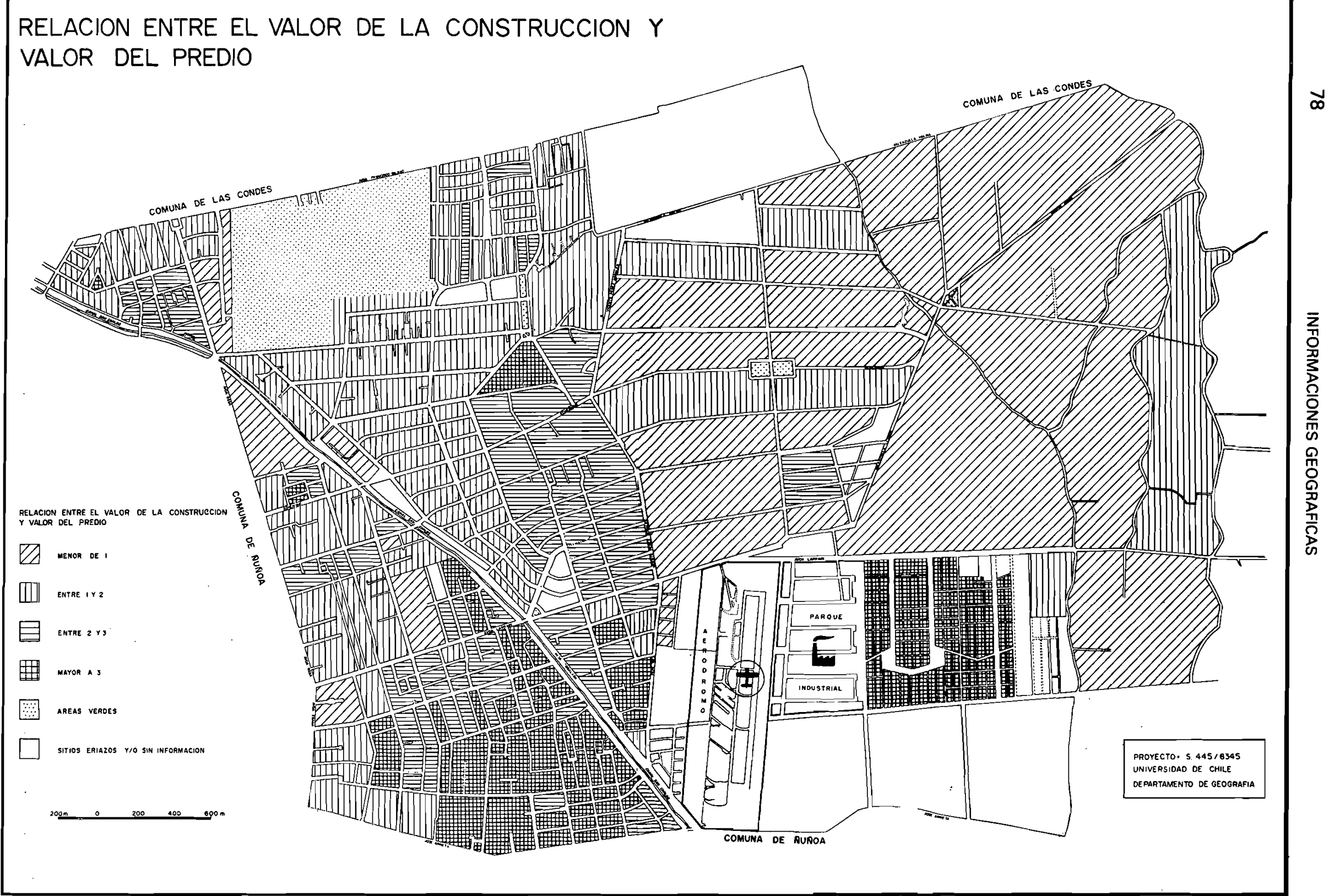


manzanas, construcciones de calidad inferior y superficie promedio de las viviendas entre 60 y 80 $\mathrm{m}^{2}$. Esta diferencia obedece a que el sector Norte, se encuentra física y funcionalmente más integrado a las tradicionales comunas orientales del Area Metropolitana de elevados status socioeconómicos, las que proyectan sus efectos valorizantes en este espacio.

\subsection{AREA URBANA DE TRANSICION-EXPANSION:}

Comprende el sector oriente de la Comuna. La ocupación del espacio es relativamente reciente, predominando las viviendas edificadas con posterioridad a la década del 60.

Dos sectores diferenciados según su uso, es posible distinguir: Area Media propiamente tal, destinada primitivamente a una función mixta de tipo residencia-agricola y Reina Alta, limitada hasta 1980 a la expansión urbana por disposiciones de administración local y cuyo uso predominante estaba basado en parcelas de agrado, de viviendas semipermanentes, para sectores de altos ingresos.

En general el área de transición-expansión, presenta una gran homogeneidad expresada en el tamaño de la vivienda (alrededor de $100 \mathrm{~m}^{2}$.); predominio de la calidad corriente de la construcción, pero a la vez, por una mayor frecuencia de calidad media-superior y superior y por el alto valor que alcanza el suelo, el que en promedio, llega a ser superior al de la construcción.

A pesar de ser el sector de menor accesibilidad o más excéntrico, se caracteriza por presentar las mayores rentas económicas desde el punto de vista del valor del suelo. Factores tales como posibilidad de construcción de viviendas unifamiliares de mayor superficie y privacidad, junto a características ambientales más óptimas como áreas verdes y aire puro (sello perceptivo que caracteriza a la Comuna) han incidido en la polarización de los grupos poblacionales de alto status económico.

\subsection{AREA URBANA PERIFERICA-CONSOLIDADA:}

Comprende el sector al Sur de la Av. Larraín. Su origen se remonta a la última década, caracterizándose por el promedio de la vivienda de calidad media inferior, de menor tamaño $\left(40 \mathrm{~m}^{2}\right.$.), por la alta densidad de viviendas por manzanas y por los bajos montos del valor del suelo. Esta área se encuentra integrada por seis poblaciones de viviendas definitivas y cuatro campamentos de viviendas transitorias, con una población de 13.280 habitantes, lo que significa que alrededor del $18 \%$ de la población de la Comuna se encuentra espacialmente segregada en este sector. En relación al conjunto comunal, aparece como una isla, limitada al poniente por el Aeródromo y el Parque Industrial, usos del suelo que inciden en la menor valorización de este espacio, en cambio hacia el Oriente, la localización de una infraestructura educacional, induce a suponer que no se incorporarán nuevas áreas al uso residencial.

Tres unidades de observación se han considerado al interior de este espacio que hemos calificado de hábitat subintegrado.

\subsubsection{Poblaciones de erradicación de Iniclativa privada y apoyo estatal:}

Su origen está ligado a organizaciones espontáneas de pobladores, sin acceso al mercado formal de la vivienda, que organizados a través de cooperativas, se consiguen el apoyo estatal a través de instituciones como la Corporación Habitacional (CORHABIT) y el Servicio de la Vivienda y Urbanismo (SERVIU), dependientes del Ministerio de la Vivienda y la acción directa del Municipio. Este es el caso de Villa La Reina, el mayor conjunto poblacional, que reúne cerca de 1.400 familias cuyo origen, está ligado a la parcelación del antiguo fundo del mismo nombre, localizado en el sector periférico de la Comuna. A fines de la década del 50 , la parcelación de dicho predio, originó el problema habitacional de ex parceleros como de diversos pobladores de la comuna que no habían solucionado el problema de la vivienda independiente. Esta organización, propone a las autoridades la compra del fundo de 207 ha. de propiedad del Servicio de Seguro Social, de las cuales 40 fueron destinadas a la construcción de la Villa por intermedio de un sistema de autoconstrucción.

De este modo, se establecieron los requisitos básicos para optar a la vivienda definitiva; libreta de ahorro y una cuota mensual destinada a la compra de materiales de construcción a través de un préstamo, solicitado a coRHABIT. El plan contemplaba la construcción de 1.592 viviendas.

La concreción de dicho proyecto, representó un original sistema de cooperación en el cual se involucraron tanto el Ministerio respectivo, la Municipalidad, como la Universidad de Chile, que a 
través del Departamento de Arquitectura y mediante prácticas de los alumnos, elaboraron gratuitamente los planos y supervisaron los aspectos técnicos y, finalmente, la acción directa de los pobladores en la autoconstrucción de sus viviendas. De esta forma, en 1973 se entregan los títulos definitivos de 1.219 viviendas. En la actualidad se concluyen las etapas finales de las 387 viviendas restantes.

La adquisición de dicho predio por la Municipalidad, le permitió continuar en la misma área con las politicas de viviendas populares, construcción de un parque industrial, y creación de la mayoria de la infraestrura educacional pública que existe en la comuna. Este hecho, un tanto al azar en la dotación de infraestructura comunal, explica la falta de racionalidad en la localización de dichos servicios, ya que en la actualidad el área presenta graves problemas de accesibilidad y congestionamiento en la Av. Larrain, la princípal arteria de comunicación de la comuna con el conjunto metropolitano.

Con un sistema similar al anterior, se concluye, hacia 1974 un segundo conjunto habitacional de 500 viviendas, también de material sólido, denominado Danilo Vicencio y Roberto Valladares.

A partir de 1974, las soluciones habitacionales para los sectores de escasos recursos adopta una modalidad que privilegia absolutamente la participación pública, a través del Estado, SERViu y de la Municipalidad, permitiendo distinguir un segundo tipo de poblaciones generadas a partir de dicha fecha.

3.3.2. Poblaciones de erradicaclón derlvadas de organismos de goblerno, insertas en las políticas habitaclonales de sectores de escasos recursos.

Del plan de Construcción de Vivienda de Emergencia por parte del serviu e implementadas por la Municipalidad, se construyen entre 1975 y 1976 las poblaciones "Las Perdices" y "Oscar Bonilla" que en conjunto reúnen 360 familias, en viviendas de material liviano, madera y bloques. Un tercer conjunto habitacional de material sólido creado recientemente en 1978 llamado "Río Lauca", 121 familias, completa el cuadro.

En general, es posible suponer un mejor nivel socioeconómico en los sectores de poblaciones que en los campamentos, considerando que los beneficiados con estas políticas habitacionales, han debido cumplir ciertos requisitos de ahorro mínimo. Cabe esperar, mejores niveles en lo que se refiere a trabajo, educación y, posiblemente en las tipologias de la estructura demográfica.

\subsubsection{Campamentos:}

Están constituidos por erradicación de familias de extrema pobreza que vivian en condiciones ilegales en diversos sectores residenciales de la comuna. Este es el caso de los campamentos Pepe Vila (1975) y Los Anhelos (1968); los dos restantes, Toqui Lautaro y Nuestra Esperanza, fueron en su origen antiguas agrupaciones espontáneas, en pequeños sitios vacíos al interior del conjunto residencial. Ante los agudos problemas de insalubridad, acentuados por el incremento de la densidad demográfica, las autoridades municipales intervienen ahora en la dotación de servicios básicos, reglamentación y ordenamiento del espacio interno.

En estas circunstancias, los habitantes se encuentran en una situación legal, pero transitoria, sin tenencia definitiva ya que deberán ser erradicados conforme a los programas de construcción de viviendas sociales que se proponen implementar las autoridades municipales.

Se caracterizan por un nivel socioeconómico bajo; las viviendas son de apariencia modestas. con alto grado de hacinamiento, dotación de infraestructura mínima y de carácter colectivo lagua - cabinas públicas) y evidentes problemas ambientales derivados de las formas de evacuación de aguas servidas, además de escasez de agua potable, y falta de pavimentación de calles y de pasajes interiores.

\section{DIFERENCIACION SOCIAL AL INTERIOR DEL HABITAT SUBINTEGRADO}

Del análisis de la encuesta del Comité de Acción Social (C.A.S.) del año 1982 en cada una de las unidades de observación, se intenta determinar el grado de diferenciación interna en cuanto al contenido socioeconómico e infraestructura de las poblaciones y campamentos.

El Cuadro 1, muestra en relación a la vivienda y al equipamiento básico tal diferenciación.

El sector de campamentos aparece como el más deprimido dentro del conjunto hábitat subintegrado, en tanto que en el caso de las poblaciones en general, no se observan diferencias significativas. Donde éstas sí resuitan más evidentes, es en relación a la actividad ocupacional de la población. A tal fin se ha utilizado el indice de segregación planteado por Taeuber en DuNCAN Y 
Cuadro 1: Hábitat Sublntegrado: variables de vivienda y equipamiento. 1982

\begin{tabular}{|c|c|c|c|c|c|c|c|c|c|}
\hline & \multirow[t]{2}{*}{$\begin{array}{c}\% \text { en relac. } \\
\text { al total } \\
\text { de famils. }\end{array}$} & \multirow[t]{2}{*}{$\begin{array}{c}\% \text { de } \\
\text { viviendas } \\
\text { tipo casa. }\end{array}$} & \multirow[t]{2}{*}{$\begin{array}{l}\% \text { de vivien- } \\
\text { das tipo } \\
\text { mediaguas. }\end{array}$} & \multicolumn{2}{|c|}{$\begin{array}{c}\text { Equipam. } \\
\text { Básico }\end{array}$} & \multicolumn{2}{|c|}{$\begin{array}{l}\text { Comb. } \\
\text { Cocinar }\end{array}$} & \multicolumn{2}{|c|}{$\begin{array}{l}\text { Analf. } \\
\text { May. } 15 \\
\text { años }\end{array}$} \\
\hline & & & & $\begin{array}{c}\text { Sin } \\
\text { agua } \\
\text { pot. } \\
\%\end{array}$ & $\begin{array}{l}\text { Sin } \\
\text { Eléc. } \\
\%\end{array}$ & $\begin{array}{l}\text { Sin } \\
\text { Alc. } \\
\%\end{array}$ & $\begin{array}{l}\text { Gas } \\
\%\end{array}$ & $\begin{array}{c}\text { Carb. } \\
\text { para- } \\
\text { fina } \\
\%\end{array}$ & $\%$ \\
\hline Población A & 67,8 & 87,9 & 11,8 & $\overline{10,2}$ & 1,3 & 15,6 & 95,1 & 4,7 & 9,1 \\
\hline Población B & 16,4 & 96,4 & 3,2 & 2,1 & 0,8 & 3,8 & 93,3 & 6,5 & 9,0 \\
\hline $\begin{array}{l}\text { Campa- } \\
\text { mentos }\end{array}$ & 15,8 & 0,8 & 98,8 & 95,7 & 6,1 & 98,8 & 60,6 & 33,7 & 14,7 \\
\hline
\end{tabular}

NOTA: El conjunto de población A, corresponde a la categoría de poblaciones de erradicación de iniciativa privada y apoyo estatal. El conjunto de población $B$, corresponde a poblaciones de erradicación derivadas de organismos de Gobierno, insertas en las políticas habitacionales de sectores de escasos recursos.

Duncan (1955), el cual tiende a medir los agrupamientos de población (en este caso, de la población actival en determinadas áreas. La fórmula para medir dicho indice es la siguiente:

Iqo $=\frac{\text { qo }}{\text { to }}-\frac{\mathrm{Q}-\mathrm{qo}}{\mathrm{T}-\mathrm{to}} \cdot 100$

Donde:

lqo $=$ Indice de segregación del grupo q en la unidad de observación o.

qo $=$ población del grupo q en la unidad de observación 0 .

to = población total activa de la unidad de observación o.

$\mathrm{Q}$ = población total activa del grupo $\mathrm{q}$ en el hábitat subintegrado.

$\mathrm{T}=$ población total activa en el hábitat subintegrado.

La definición planteada por BOAL (1976), es que un grupo particular no presenta segregación cuando sus miembros están distribuidos de manera uniforme con respecto al resto de la población. Cualquiera desviación de tal uniformidad, representa una situación caracterizàda por segregación.

El Cuadro 2 señala en sus valores absolutos las actividades desempeñadas por la población en las diferentes categorias de ocupación.

Cuadro 2: Actividad Ocupacional de la población del hábitat Subintegrado. 1982

\begin{tabular}{|c|c|c|c|c|c|c|c|c|c|c|}
\hline & $\begin{array}{c}\text { (1) } \\
\text { Prof. } \\
\text { Empls. }\end{array}$ & $\begin{array}{c}\text { (2) } \\
\text { Jubil. } \\
\text { Pens. }\end{array}$ & $\begin{array}{c}\text { (3) } \\
\text { Obrs. }\end{array}$ & $\begin{array}{c}\text { (4) } \\
\text { Serv. } \\
\text { Mens. }\end{array}$ & $\begin{array}{c}\text { (5) } \\
\text { Jarn. }\end{array}$ & $\begin{array}{c}\text { (6) } \\
\text { Comer. } \\
\text { Ambul. }\end{array}$ & $\begin{array}{l}\text { (7) } \\
\text { Ces. }\end{array}$ & $\begin{array}{c}\text { (8) } \\
\text { PEM. }\end{array}$ & $\begin{array}{l}\text { (9) } \\
\text { Otrs. }\end{array}$ & Total \\
\hline Población A & 389 & 239 & 949 & 31 & 202 & 136 & 161 & 4 & 70 & 2.181 \\
\hline Población B & 81 & 30 & 242 & 3 & 72 & 34 & 45 & 2 & 18 & 527 \\
\hline $\begin{array}{l}\text { Campa- } \\
\text { mentos }\end{array}$ & 23 & 26 & 211 & 5 & 122 & 44 & 68 & 4 & 7 & 510 \\
\hline Total & 493 & 295 & 1.402 & 39 & 396 & 214 & 274 & 10 & 95 & 3.218 \\
\hline
\end{tabular}

Aplicado el índice de Taeuber se obtuvo los siguientes valores para los grupos en cuestión. los cuales se señalan en en Cuadro 3.

Al respecto es riecesario señalar que un valor negativo indica que la proporción es más fuerte en el conjunto de las unidades de observación que en la unidad considerada; en cambio, un valor positivo expresa que la proporción es más fuerte en la unidad de observación. 
Cuadro 3: Indices de Segregación Ocupacional. 1982.

\begin{tabular}{crrrrrrrrr}
\hline & & \multicolumn{8}{c}{ Actividad Ocupacional } \\
& \multicolumn{1}{c}{$(1)$} & \multicolumn{1}{c}{$(2)$} & \multicolumn{1}{c}{$(3)$} & \multicolumn{1}{c}{$(4)$} & \multicolumn{1}{c}{$(5)$} & \multicolumn{1}{c}{$(6)$} & \multicolumn{1}{c}{$(7)$} & \multicolumn{1}{c}{$(8)$} & \multicolumn{1}{c}{$(9)$} \\
\hline Población A & 7.8 & 5.5 & -0.1 & 2.1 & -9.5 & -1.3 & -3.5 & -0.4 & 8.0 \\
Población B & 0.0 & -4.0 & 2.8 & -0.8 & 1.6 & -0.2 & 0.0 & 0.1 & 0.6 \\
Campamentos & -12.8 & -4.9 & -2.6 & -0.3 & 13.8 & 2.4 & 5.7 & 0.5 & -1.9 \\
\hline
\end{tabular}

El patrón resultante de considerar la actividad ocupacional de la población económicamente activa al interior del hábitat subintegrado, muestra que profesionales se encuentran especialmente segregados en asentamientos de tipo $A$, en tanto, que en campamentos se concentra un mayor porcentaje de personas en los extremos inferiores de la jerarquía ocupacional, sobresaliendo por sus altos índices jornaleros y cesantes $(13,8 \%$ y $5,7 \%$ respectivamente). Aspectos relacionados al nivel cultural de la población y al origen de cada asentamiento, entre otros, surgen como variables explicativas de tal diferenciación al interior de la aparente homogeneidad de este espacio social.

\section{CONCLUSIONES}

A partir de esta serie de características físicas de la vivienda se han podido identificar patrones espaciales referidos al contenido socioeconómico al interior del espacio comunal. No obstante, para implementar los pasos futuros que se persiguen en esta investigación, se hace imprescindible contrastar dichos patrones con variables demográficas, económicas, sociales y culturales, las cuales tradicionalmente han ejercido un alto peso en la diferenciación del espacio social, a la vez que permiten explicar con mayor exactitud los aspectos dinámicos que están presentes en la generación de tales estructuras.

El actual proceso de expansión muestra que la comuna tiende a consolidarse, desde el punto de vista residencial, como un sector de altos ingresos en aquellos sectores donde la disponibilidad de suelo urbano posibilita el aumento de la densidad residencial, proceso que unido a la existencia de áreas fuertemente consolidadas de bajo status, tiende a acentuar la diferenciación socioespacial del territorio comunal.

Finalmente, el grado de integración y adaptación funcional de la población al interior de la aparente homogeneidad social del hábitat subintegrado, en términos de trabajo, comercio y servicios, representa las problemáticas que dirigirán el curso futuro de esta invstigación. De esta manera, la determinación de estructuras, representa sólo un antecedente preliminar para avanzar en el conocimiento del sistema intraurbano.

\section{BIBLIOGRAFIA}

Berry, B. 1975. Comparative factorial ecology. Economic Geogr. 47 (2) 1 pp.

Berry, B. 1977. Contemporary urban ecology. Macmillan New York.

BoAL, F. 1976. Ethnic residential segretation. En: Herbert J.T., R.J. Johston (Eds.) Spatial Process and Form. Wiley, New York: $41-79$

Buttimer, A. 1982. Apreendendo o dinamismo do mundo vivido. En: A. Chritofoletti (Ed.) Perspectivas da Geografia. DifEL, Sao Paulo: 165-193.

Duncan, O. y B. Duncan. 1955. A methodological analysis of segregation indices. Amer. 20: 210-217.

Durán, C. 1982. La Reina: Planeamiento y Acción Privada. Facultad de Arquitectura y Urbanismo, Universidad de Chile. Santiago.

Herbert, D. 1972. Urban geography a social perspective. Newton Abbot, Devon, David and Charles.

HeRbert, D. 1979. Social problems and the city; geographical perspectives. Oxford Univ. Press.

Heraert, D. y R. Johnston. 1978. Social areas in cities. Process, patterns and problems. Chichester, Wiley. N.Y.

Instituto Nacional de Estadísticas, 1982. XV Censo Nacional de Población y IV de Vivienda. Cifras Preliminares. Santiago.

1. Municipalidad de La Reina. 1982. Encuesta de Acción Social (CAS).

SABATINI, F. 1981. La dimensión ambiental de la pobreza urbana en la teorías latinoamericanas de marginalidad. EURE, 3 (23): 53-67.

Servicio de Impuestos Internos. 1980. Catastro Urbano. Santiago. 